\title{
The Effects of Crosslinkers on Physical, Mechanical, and Cytotoxic Properties of Gelatin Sponge Prepared via in-situ Gas Foaming Method as a Tissue Engineering Scaffold
}

S. Ali Poursamar ${ }^{\mathrm{a}}$, Alexander N. Lehner ${ }^{\mathrm{b}}$, Mahmoud Azamic ${ }^{\mathrm{c}}$, Somayeh Ebrahimi-Barough ${ }^{\mathrm{c}}$, Ali Samadikuchaksaraei ${ }^{\mathrm{de}, \mathrm{f}}$, A.P.M. Antunes ${ }^{\mathrm{a}}$

\footnotetext{
a Institute for Creative Leather Technologies, Park Campus, The University of Northampton, Boughton Green Road, Northampton, NN2 7AL, UK.,

${ }^{\mathrm{b}}$ Centre for Physical Activity and Chronic Disease and the Aging Research Centre, Institute for Health and Wellbeing, School of Health, Park Campus, The University of Northampton, Boughton Green Road, Northampton, NN2 7AL, UK.,

${ }^{\mathrm{c}}$ Department of Tissue Engineering, School of Advanced Technologies in Medicine, Tehran University of Medical Sciences, Tehran, Iran,

${ }^{\mathrm{d}}$ Cellular and Molecular Research Centre, Iran University of Medical Sciences, Tehran, Iran

e Department of Tissue Engineering \& Regenerative Medicine, Faculty of Advanced Technologies in Medicine, Iran University of Medical Sciences, Tehran, Iran.

${ }_{\mathrm{f}}^{\mathrm{f}}$ Department of Medical Biotechnology, Faculty of Allied Medicine, Iran University of Medical Sciences, Tehran, Iran.
} 
Abstract: In this study porous gelatin scaffolds were prepared using in-situ gas foaming, and four crosslinking agents were used to determine a biocompatible and effective crosslinker that is suitable for such a method. Crosslinkers used in this study included: hexamethylene diisocyanate (HMDI), poly(ethylene glycol) diglycidyl ether (Epoxy), glutaraldehyde (GTA), and genipin. The prepared porous structures were analyzed using Fourier Transform Infrared Spectroscopy (FT-IR), thermal and mechanical analysis as well as water absorption analysis. The microstructures of the prepared samples were analyzed using Scanning Electron Microscopy (SEM). The effects of the crosslinking agents were studied on the cytotoxicity of the porous structure indirectly using MTT analysis. The affinity of L929 mouse fibroblast cells for attachment on the scaffold surfaces was investigated by direct cell seeding and DAPI-staining technique. It was shown that while all of the studied crosslinking agents were capable of stabilizing prepared gelatin scaffolds, there are noticeable differences among physical and mechanical properties of samples based on the crosslinker type. Epoxycrosslinked scaffolds showed a higher capacity for water absorption and better microstructures than the rest of crosslinked samples, whereas genipin and GTA-crosslinked scaffolds demonstrated higher mechanical strength. Cytotoxicity analysis showed the superior biocompatibility of the naturally occurring genipin in comparison with other synthetic crosslinking agents, in particular relative to GTA-crosslinked samples.

Keywords: Gas foaming; Denaturation temperature; Over-crosslinking; Natural crosslinker;

\section{Introduction}

Gelatin has a remarkable foaming ability and can therefore be processed using gas foaming into porous structures without requiring additional surfactants and foaming agents [1]. The in-situ gas foaming method is a simple, non-expensive, and effective variant of the gas foaming technique [2]. It was shown that crosslinking is an important element for 
successful manufacturing of gelatin scaffolds via in-situ gas foaming and is critical in preserving the porous structure of fabricated scaffolds [3]. Different crosslinking agents have been used to stabilize gelatin tissue engineering scaffolds, four of which are the focus of this study; GTA, hexamethylene diisocyanate (HMDI), and poly(ethylene glycol) diglycidyl ether (Epoxy). Figure 1 shows the molecular structures of crosslinking agents used in this study. GTA is one of the most reported crosslinking agents in the biomedical field owing to its demonstrated effectiveness, despite being known to elicit cytotoxicity [4,5]. GTA has been applied for processing certain xenograft implants such as bioprosthetic heart valves or surgical sealants [6,7]. The GTA reaction mechanism is described as a Schiff base reaction. As a part of Schiff base reactions, carbon and nitrogen double bonds $(\mathrm{C}=\mathrm{N})$ are established between GTA and the amine groups of Lysine in gelatin molecules [8].<smiles>COC(=O)C1=CO[C@@H](O)[C@H]2CC=C(CO)[C@H]12</smiles>

Genipin<smiles>O=CCCCC=O</smiles>

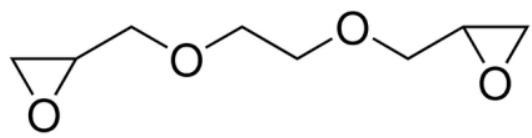

Poly(ethylene glycol) diglycidyl ether<smiles>O=C=NCCCCCCCN=C=O</smiles>

Hexamethylene diisocyanate

Figure 1: Molecular structures of crosslinkers used in this study.

As an alternative to synthetic crosslinkers such as GTA naturally occurring crosslinking agents such as genipin have been used. Genipin is extracted from the fruits of plants that are native to South America and South East Asia and has been used in Chinese herbal medicine 
[9]. With less toxicity relative to GTA, genipin reacts with amino-containing materials and has been used in crosslinking gelatin microcapsules for drug delivery, conduits for peripheral nerve regeneration, and composite for Guided Bone Regeneration [10].

HMDI molecules with two cyanate groups react either with amine groups or hydroxyl groups of gelatin to form urea or urethane bonds, respectively. HMDI has been reported as a crosslinking agent for applications in bone and cartilage tissue engineering [11], and as a coupling agent for the surface treatment of metallic and ceramic materials $[12,13]$. The driving force behind the crosslinking reaction that involves epoxy molecules is the strained covalent bonds that compose three atom ether rings at both ends of poly(ethylene glycol) diglycidyl ether. One practical advantage of epoxy compounds is their ability in reacting with a variety of functional groups (this includes carboxylic, amine, and hydroxyl groups) at a wide range of $\mathrm{pH}$ 's [14]. Figure 2 summarizes the reaction mechanisms of applied crosslinking agents. 

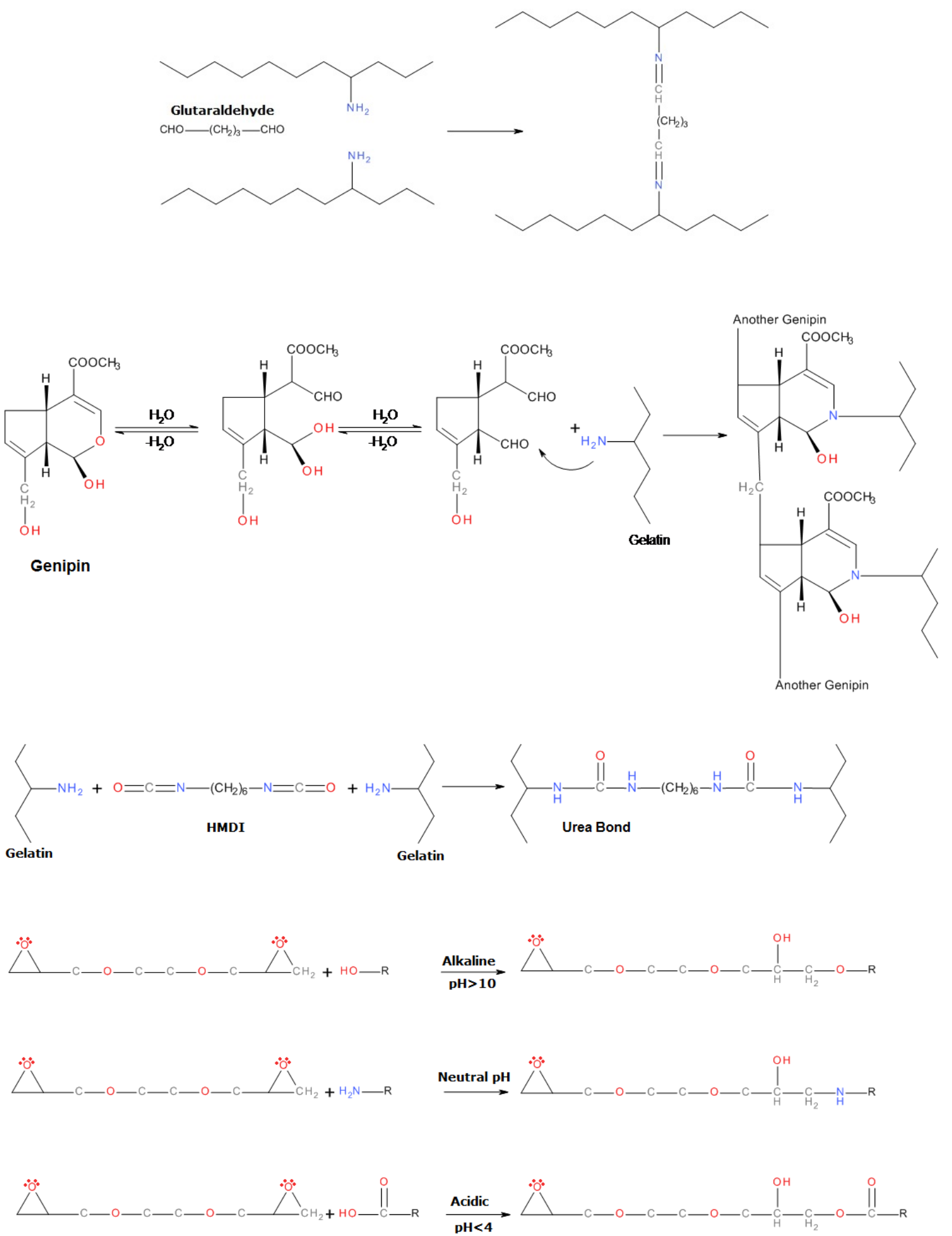

Figure 2: The summary of reaction mechanisms for the crosslinking agents used in this study. All of the applied crosslinking agents either can react with amine functional groups of gelatin exclusively or the reaction with amine functional groups is one of their possible reaction mechanism with gelatin molecules. 
In our previous study the application of crosslinking agents proved to be an essential part of in-situ gas foaming [3]. Although crosslinking has a deep impact on the mechanical and physical properties of samples prepared via in-situ gas foaming there is no comprehensive study that identifies the optimum crosslinking agent for application in this method, to the best of the authors' knowledge. In addition this study strives to add to the relatively limited number of reports on HMDI application as a crosslinker for gelatin samples.

Here, a comparative study is reported with the aim of identifying a biocompatible and effective crosslinker for application in in-situ gas foaming. The properties of prepared samples were characterized using a Scanning Electron Microscope (SEM), Fourier Transform Infrared spectroscopy, thermal analysis, tensile strength analysis, and water absorption analysis. To compare the biocompatibility of the samples L929 fibroblast cells were cultured, stained, and fixed to study their spreading and attachment using confocal and scanning electron microscopes.

\section{Materials and Methods}

\subsection{Scaffold Preparation Method}

Porous gelatin scaffolds were prepared according to the method described earlier with some modifications [3]. Briefly, type B gelatin powder (Sigma Aldrich, USA) was used to prepare a $20 \% \mathrm{w} / \mathrm{v}$ aqueous solution. Sodium bicarbonate (BDH Chemical, UK) was directly added to the gelatin solution. Consequently, a $360 \mu 1$ aliquot of acetic acid (Fisher Scientific, UK) was added to initiate the foaming process. The gelatin foam was cast in the polystyrene molds, $5.5 \mathrm{~cm}$ in diameter, and frozen for 1 hour at $-25^{\circ} \mathrm{C}$. The frozen foam blocks were then extracted from the molds and incubated in $4^{\circ} \mathrm{C}$ de-ionised water to extract unreacted components. The preparation process was continued with the samples crosslinking as follows: 


\section{Crosslinking Methods}

GTA Crosslinking: Upon removal from $4^{\circ} \mathrm{C}$ de-ionised water, the samples were incubated in aqueous solutions of GTA for 3 hours. GTA aqueous solutions were prepared from a $50 \% \mathrm{v} / \mathrm{v}$ aqueous stock solution of GTA (Fisher Scientific, UK). An aliquot of $500 \mu \mathrm{l}$ GTA was added to $50 \mathrm{ml}$ de-ionised water providing $0.5 \mathrm{~mol} / \mathrm{v}$ GTA solution.

Epoxy Crosslinking: An aliquot of $1.15 \mathrm{ml}$ poly(ethylene glycol) diglycidyl (Sigma Life Science, Japan) was added to $50 \mathrm{ml}$ of de-ionised water making $0.5 \mathrm{~mol} / \mathrm{v}$ solution. Upon removal from $4^{\circ} \mathrm{C}$ de-ionised water, the samples were incubated in the prepared epoxy solution for 3 hours.

HMDI Crosslinking: Dehydration of samples and crosslinking of scaffolds with HMDI was performed according to the method reported by Catalina et al. (2011). The samples were dehydrated in a gradient of propan-2-ol aqueous solutions of 25, 50, 75, and $100 \% \mathrm{v} / \mathrm{v}$ (each step 30 minutes under agitation). Consequently, the scaffolds were incubated in propan-2-ol solution of $\mathrm{HMDI}$ at $4^{\circ} \mathrm{C}$ for 16 hours. To prepare the HMDI solution, $400 \mu \mathrm{l}$ of HMDI was added to $50 \mathrm{ml}$ of propon-2-ol giving $0.5 \mathrm{~mol} / \mathrm{v}$ concentration. After crosslinking, the samples were re-hydrated in a gradient of propan-2-ol aqueous solutions in the reverse order used in pre-crosslinking preparation (i.e. $100,75,50,25 \% \mathrm{v} / \mathrm{v}$ propan-2-ol aqueous solutions).

Genipin Crosslinking: The crosslinking of samples was performed according to the method reported by Bigi et al. [15]. Soaked samples were incubated in Phosphate Buffered Solution (PBS) of genipin for 14 hours at room temperature without agitation. To prepare the crosslinking solution, 1.13 grams of genipin (Challenge Bioproducts, Taiwan) was added to $100 \mathrm{ml}$ of PBS solution providing a genipin solution with concentration of $0.5 \mathrm{~mol} / \mathrm{v}$. 
Upon crosslinking, the samples were washed in de-ionised water overnight, frozen, and lyophilised at $-40^{\circ} \mathrm{C}$ and under a vacuum pressure of 0.250 mbar for 1 day. Non-crosslinked samples were prepared for comparison by removing the crosslinking step from the process.

\subsection{Characterization of the Prepared Scaffolds}

\subsubsection{Mechanical Properties}

Once the samples were conditioned at 95\% relative humidity for 2 days, the mechanical testing was carried out using a texture analyzer (TA.XT-Plus, Stable Micro Systems, UK). Samples were cut into rectangular strips $(10 \times 5 \mathrm{~mm})$, thicknesses were measured at 3 points, and the average value was recorded. The samples were drawn at a cross head speed of $0.033 \mathrm{~mm} \cdot \mathrm{sec}^{-1}$. The tensile strength and strain values are reported in $\mathrm{kPa}$ and percentage $(\%)$, respectively. The Young's modulus of the scaffolds were calculated as the slope of the linear segment of Stress - Strain curve and are reported in $\mathrm{kPa}$. The tests were performed in triplicate.

\subsubsection{Thermal Analysis}

The samples were conditioned at $65 \%$ relative humidity for 2 days prior to analysis. Differential Scanning Calorimetry (DSC - 822e, Mettler-Toledo, Switzerland) was used for thermal analysis. The samples were sealed in $40 \mu$ l aluminium pans and heated from 15 to $100^{\circ} \mathrm{C}$ at a heating rate of $5^{\circ} \mathrm{C} \cdot \mathrm{min}^{-1}$. The peak temperature and the normalized enthalpy of transition of each sample were recorded. The peak temperature was assigned as the gelatin denaturation temperature $\left(\mathrm{T}_{\mathrm{d}}\right)$. The experiments were performed in triplicate.

\subsubsection{Fourier Transform Infra-Red Spectroscopy (FT-IR)}

Fourier Transform Infra-Red spectroscopy (FTIR/ATR-4800s, Shimadzu, Japan) was performed by scanning from 4000 to $1000 \mathrm{~cm}^{-1}$ at a nominal resolution of $4 \mathrm{~cm}^{-1}$ using 264 scans. Multiple scans were performed on each sample and a representative FT-IR diagram for each sample is chosen for presentation. 


\subsubsection{Swelling Ratio}

The samples were conditioned in a $0 \%$ relative humidity desiccator for 2 days. Dry samples were weighed and incubated in de-ionised water. To prevent non-crosslinked sample disintegration in water during analysis, the samples were kept at $4{ }^{\circ} \mathrm{C}$ (below gelling point of gelatin). The hydrated samples were removed from water at intervals of 1, 3, and 6 hours. Removal of excess superficial water with filter paper was carried out and the sample weights were recorded. Measurement for each batch of samples was carried out in duplicate and the average value of the two results is reported. The swelling ratio was calculated using Equation 1 , where $\mathrm{W}_{\mathrm{H}}$ and $\mathrm{W}_{\mathrm{D}}$ are the sample hydrated weight and initial dry weight, respectively.

$$
\text { Swelling Ratio } \left.(\mathrm{g} / \mathrm{g} \%)=\left[\left(\mathrm{W}_{\mathrm{H}}-\mathrm{W}_{\mathrm{D}}\right) / \mathrm{W}_{\mathrm{D}}\right] \times 100 \quad \text { (Equation } 1\right)
$$

\subsubsection{Microstructure Analysis}

The scaffolds microstructure was examined using a scanning electron microscope (S3000N, Hitachi, Japan). Samples were gold-coated using a sputter coater (SC500, Mscope, UK). The average pore sizes of the scaffolds were determined using Quartz PCI image processing software package (Quartz Image Corp., Vancouver, Canada).

\subsubsection{Cell Culture Studies}

Cell culture studies were conducted using L929 mouse fibroblast cell line (Pasteur Institute, Iran) as cultured in Dulbecco's Modified Eagle Medium (DMEM, Gibco, USA) supplemented with 10\% Fetal Bovine Serum (FBS) and $50 \mathrm{U} / \mathrm{ml}$ penicillin and $50 \mathrm{U} / \mathrm{ml}$ streptomycin at $37^{\circ} \mathrm{C}$ with $5 \% \mathrm{CO}_{2}$.

After fibroblast cells reached a sub-confluent state of $70 \%$, they were trypsinised by $0.25 \%$ trypsin/EDTA for 5 minutes at $37^{\circ} \mathrm{C}$. The scaffolds were cut into small pieces and sterilised with $70 \%$ ethanol for 2 hours and then under ultraviolet light overnight. Trypsinised cells were seeded on the specimens with a final seeding density of $5 \times 10^{4}$ cells/scaffold. 
Cellular viability was measured indirectly using MTT assay at the first, third, and fifth days of the cell culture experiments. The assay reflected the activity of a mitochondrial dehydrogenase that transforms light yellow MTT into dark blue Formazan. From a solution of 3-[4,5-dimethylthiazol-2-yl]-2,5-diphenyltetrazolium bromide (MTT) with concentration of $5 \mathrm{mg} / \mathrm{ml}$, a $40 \mu 1$ aliquot was added to each well, and the plates were incubated at $37^{\circ} \mathrm{C}$ for 4 hours. The medium was removed and the formazan crystals dissolved in DMSO. The absorption of the formazan solution was measured at $570 \mathrm{~nm}$ using an ELISA reader (Expert 96, Asys Hitch, Austria). The experiments were performed in triplicate.

To observe the spreading pattern of cultured cells after 5 days of incubation, SEM and DAPI-staining techniques were used. The scaffolds were washed with PBS and the cultured cells were fixed with $2.5 \% \mathrm{v} / \mathrm{v}$ GTA solution for 1 hour, followed by washing with PBS, dehydration in gradient ethanol solutions $(30,50,70,80,90$ and $100 \%$ each lasted for 10 minutes), and lyophilisation. Dried samples were gold sputter-coated and observed using a SEM (XL-30, Philips, Netherlands), operated at $15 \mathrm{kV}$. The cultured cells nuclei were stained with 4',6'-diamidino-2-phenylindole (DAPI) to assess cell viability. The images of stained cells were captured using confocal fluorescent microscope (BX61, Olympus, Japan).

\subsubsection{Statistical Analysis}

Kruskal-Wallis method using SPSS Statistics software (Ver. 20, IBM, USA) was used to show the presence of any significant differences between the results. Pairewise tests were carried out between the crosslinked and non-crosslinked samples and differences were accepted as significant at $\mathrm{p} \leq 0.05$.

\section{Results and Discussion}

\subsection{Mechanical Properties}

Table 1 lists the mechanical properties of non-crosslinked and crosslinked samples. Among crosslinked samples GTA-crosslinked scaffolds were the strongest and had the tensile 
strength of $239.48 \mathrm{kPa}$, a significantly higher value compared with the non-crosslinked samples $(\mathrm{p}=0.05)$. Genipin-crosslinked samples had a Young's modulus of $9.3 \mathrm{kPa}$ which is higher than the Young's modulus values for the rest of tested samples and significantly higher than non-crosslinked samples $(\mathrm{p}=0.05)$. HMDI-crosslinked samples showed the highest (101\%), while genipin-crosslinked scaffolds had the lowest tensile strain $(15.7 \%)$ than the rest of crosslinked samples and its tensile strain was significantly lower than noncrosslinked samples $(\mathrm{p}=0.05)$.

Table 1: The mechanical properties of gelatin scaffolds crosslinked with different types of crosslinking agents are compared with non-crosslinked samples. GTA-crosslinked samples had higher tensile strength than the other crosslinked samples and with significantly difference with non-crosslinked samples $(p=0.05)$. Genipin-crosslinked samples showed significantly lower tensile strain and HMDI-crosslinked samples had significantly higher tensile strain relative to non-crosslinked samples $(\mathrm{p} \leq 0.05) . *$ denotes presence of significant difference between the recorded value and the non-crosslinked samples.

\begin{tabular}{|c|c|c|c|}
\hline Samples & $\begin{array}{c}\text { Tensile Strength } \\
(\mathbf{k P a})\end{array}$ & $\begin{array}{c}\text { Young's Modulus } \\
\mathbf{( k P a )}\end{array}$ & $\begin{array}{c}\text { Tensile Strain } \\
\mathbf{( \% )}\end{array}$ \\
\hline Non-Crosslinked & $80.76( \pm 4)$ & $0.87( \pm 0.1)$ & $114.83( \pm 9)$ \\
\hline Genipin & $130.8( \pm 25)$ & $9.3( \pm 1)$ & $15.7( \pm 2)^{*}$ \\
\hline GTA & $239.48( \pm 70)^{*}$ & $2.44( \pm 0.4)$ & $30.23( \pm 5)$ \\
\hline HMDI & $157.5( \pm 13)$ & $2.6( \pm 0.4)$ & $101.2( \pm 16)^{*}$ \\
\hline Epoxy & $20.3( \pm 10)$ & $0.5( \pm 0.2)$ & $53.9( \pm 16)$ \\
\hline
\end{tabular}

Different mechanical behaviours of tested samples may be explained according to the differences between the applied crosslinking agents molecular structure and their reactivity. It is reported that the molecular chain length of crosslinking agents can impact the elasticity [16], brittleness [17], and crosslinking density (index) [18] of structures. The longer crosslinker molecules can cause more flexibility in the final structure [17] and on the contrary shorter crosslinkers can bring the polymeric network closer together making it less flexible. 
Shorter crosslinked bridges between gelatin molecules are capable of establishing higher crosslinking density which can result in higher tensile strength [18]. This relation between molecular structure and tensile strength can be observed in the obtained results of GTA, HMDI, and Epoxy-crosslinked samples as shown in Table 1. However, genipin has a shorter molecular structure in comparison with the rest of crosslinking agents, but since it has lower reactivity than HMDI and GTA the tensile strength of Genipin-crosslinked scaffolds is ranked third after GTA- and HMDI-crosslinked samples in Table 1. Lower reactivity of genipin might be due to its bulky heterocyclic structure as it is reported by HC. Liang et al [19].

Epoxy-crosslinked samples showed significantly lower tensile strength not only in comparison with the other crosslinked samples but also relative to the non-crosslinked scaffolds $(\mathrm{p}=0.05)$. Reduction in tensile strength as a result of epoxy crosslinking has been reported by other researchers for both synthetic and natural polymer samples [20,21]. It has been reported that upon absorbing water, the mechanical strength of epoxy-crosslinked samples deteriorate rapidly [22]. This is thought to be due to water molecules establishing hydrogen bonds with polar functional groups of epoxy polymers, such as hydroxyl groups [23]. The crosslinking with epoxy noticeably increases the number of hydroxyl functional groups in the polymer structure (refer to Leach et al. 2005 for details of epoxy chemical reactions with proteins). This may explain the recorded reduction of mechanical strength in the epoxy-crosslinked samples, as they were conditioned at $95 \%$ relative humidity prior to testing.

\subsection{Thermal Analysis}

Table 2 shows the thermal characteristics of the prepared porous scaffolds crosslinked with different crosslinking agents and compared with non-crosslinked samples. Upon completion of crosslinking reactions, the denaturation temperature of scaffolds increased 
significantly in comparison with the non-crosslinked samples $(\mathrm{p} \leq 0.05)$. The denaturation temperature is an indirect measurement of crosslinking degree, with higher denaturation temperatures showing a greater degree of crosslinking or stabilisation [24]. This temperature is usually associated with the loss of protein activity through unfolding [25]. Shorter crosslinking agents would lead to a higher crosslinking density and a tighter polymeric network structure. Closer gelatin molecules will have less axial separation, thus more energy would be needed to unfold the protein structure and therefore require higher temperatures as observed by a higher denaturation temperature [26]. This justifies the reported higher denaturation temperature in structures that are crosslinked with shorter crosslinking agents [27]. It may also explain the higher values of denaturation temperature for scaffolds crosslinked with genipin and GTA relative to the two other crosslinking agents.

Table 2: The thermal stability of crosslinked and non-crosslinked scaffolds. Relative to the non-crosslinked samples, the denaturation temperature of gelatin was significantly increased after crosslinking regardless of used crosslinkers $(p \leq 0.05)$. Similarly, the negative values of enthalpy of transition were reduced after crosslinking regardless of applied crosslinkers. But only the enthalpy of transition in genipin-crosslinked samples was significantly lower than the non-crosslinked samples $(\mathrm{p}=0.05) .{ }^{*}$ denotes presence of significant difference between the recorded value and the non-crosslinked samples.

\begin{tabular}{|c|c|c|}
\hline Samples & $\begin{array}{c}\left.\text { Denaturation Temperature } \mathbf{( T}_{\mathbf{d}}\right) \\
\left({ }^{\mathbf{C})}\right.\end{array}$ & $\begin{array}{c}\text { Enthalpy of Transition } \\
\left(\mathbf{J . g}^{-1}\right)\end{array}$ \\
\hline Non-Crosslinked & $48.1( \pm 6.9)$ & $-25.7( \pm 16.7)$ \\
\hline Genipin & $87.6( \pm 0.3)^{*}$ & $-12.0( \pm 0.2)^{*}$ \\
\hline GTA & $84.5( \pm 1.5)^{*}$ & $-16.3( \pm 0.9)$ \\
\hline HMDI & $77.8( \pm 1.0)^{*}$ & $-15.4( \pm 0.6)$ \\
\hline Epoxy & $80.4( \pm 2.3)^{*}$ & $-19.1( \pm 0.9)$ \\
\hline
\end{tabular}

The negative values of the enthalpy of transition were lower for all of the studied crosslinked samples comparing to the non-crosslinked one. The lower value may be due to a 
reduction in the entropy of transition [28] and a reduction in the number of hydrogen bonds in favour of an increase in the number of covalent bonds after crosslinking [29].

\subsection{Fourier Transform Infra-Red Spectroscopy (FT-IR)}

Figure 3 shows the FT-IR spectra of the crosslinked scaffolds as compared with noncrosslinked samples. All spectra showed the signature FT-IR absorption bands of gelatin close to $1630,1539,1237 \mathrm{~cm}^{-1}$ which are known as amide I, II, and III, respectively. In addition all samples showed the amide A and B absorptions at the vicinity of 3298 and 3075 $\mathrm{cm}^{-1}$. The interactions between carbon and oxygen atoms as part of carbonyl groups $(\mathrm{C}=\mathrm{O})$ cause the Amide I absorption [30]. Amide II and III bands originate from N-H bending and $\mathrm{N}=\mathrm{C}$ stretching from amide linkages, although vibrations associated with amide III absorption are relatively weaker [31]. Finally, amide A and amide B are assigned to the vibrations of hydroxyl groups and N-H stretching vibrations, respectively [32].

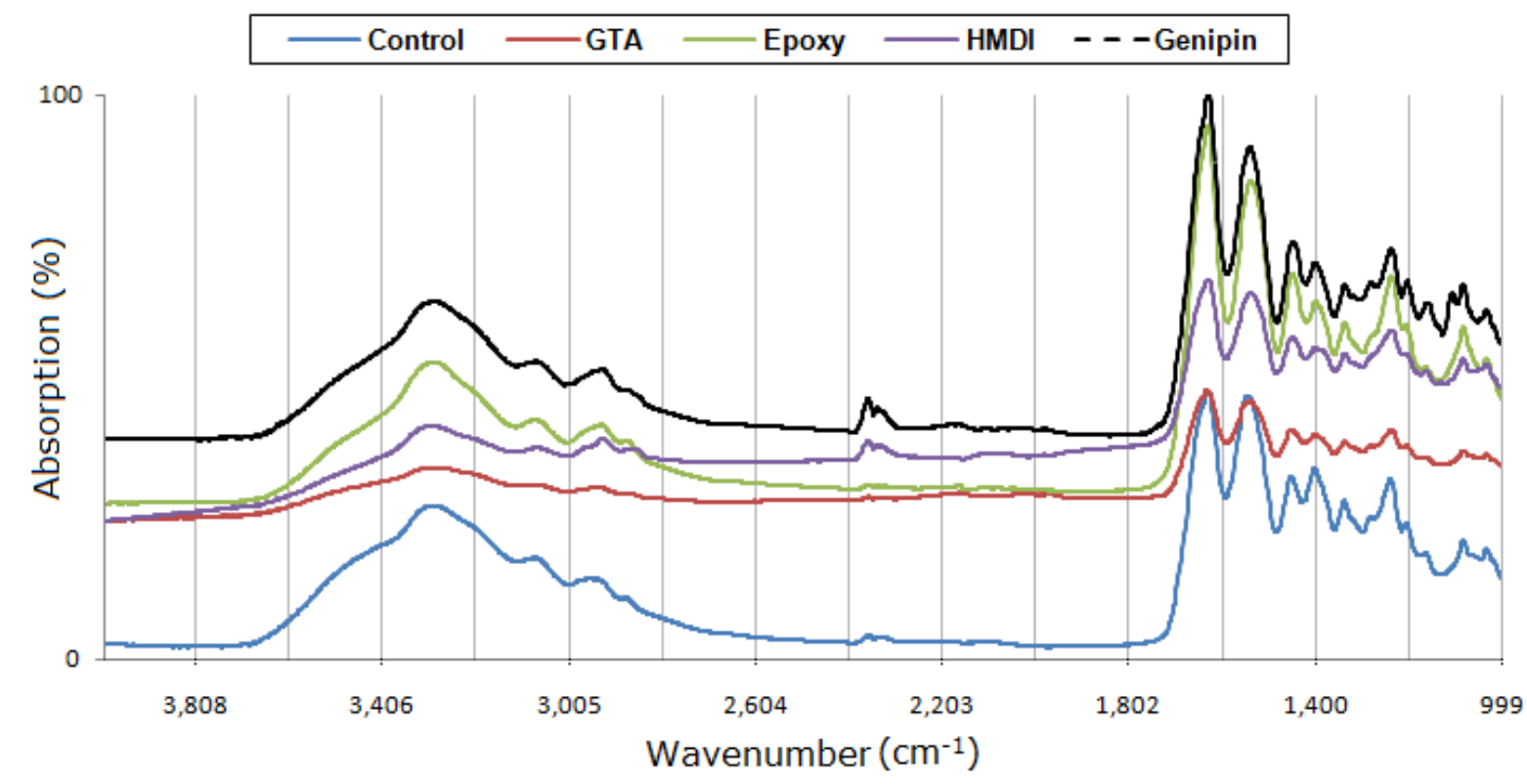

Figure 3: The FT-IR spectra of gelatin scaffolds crosslinked with four different crosslinking agents as compared with non-crosslinked samples. All spectra showed the Amide absorption bands of gelatin. Epoxy, HMDI, and Genipin spectra showed similar absorption bands at $1080 \mathrm{~cm}^{-1}$ which set them apart from GTA-crosslinked samples. 
Crosslinking with HMDI, Epoxy, and genipin had an impact on the gelatin absorption at $1080 \mathrm{~cm}^{-1}$. This absorption in the gelatin FTIR spectrum originated from the C-O stretching band [32]. Crosslinking with HMDI caused a shift of this absorption towards lower wave number $\left(1077 \mathrm{~cm}^{-1}\right)$. This absorption corresponds with the formation of urethane bonds that originated from the reaction of the isocyanate and hydroxyl functional groups [13]. In the FT-IR spectrum of genipin-crosslinked scaffolds the absorption bands close to $1100 \mathrm{~cm}^{-1}$ appeared. This absorption originates from $\mathrm{C}-\mathrm{O}-\mathrm{C}$ stretching of the genipin ring [33]. The absence of an absorption band at $2260 \mathrm{~cm}^{-1}$ in the HMDI-crosslinked scaffolds confirms no existence of the unreacted isocyanate group which is crucial for biocompatibility of the samples [34].

\subsection{Swelling Ratio}

Figure 4 compares the swelling ratio of the crosslinked gelatin porous scaffolds with non-crosslinked samples. The water absorption ability of scaffolds was significantly reduced as a result of crosslinking regardless of the crosslinker used $(\mathrm{p} \leq 0.05)$. This may be due to a reduction in the number of available free amine functional groups in the gelatin after crosslinking; these free functional groups act as sites for hydrogen bonding for water molecules [35]. The primary target for reactions of all crosslinking agents used in this study was amine groups (Figure 2). The aldehyde groups of GTA react with the amine functional groups available in lysine or hydroxylysine amino acid residues of gelatin to form a carbon and nitrogen double bond $(\mathrm{C}=\mathrm{N})$ as part of a Schiff base reaction [8]. Cyanate groups available in HMDI can react with amine groups in gelatin to form Urea bonds [13]. Epoxide functional groups may react with amine functional groups but can also react with carboxylic or hydroxyl functional groups [14]. Finally, genipin with a hemi-acetal skeleton becomes structurally equivalent to a dialdehyde by forming two aldehydic functional groups, groups which would consequently react readily with the free amine groups in gelatin [36]. 


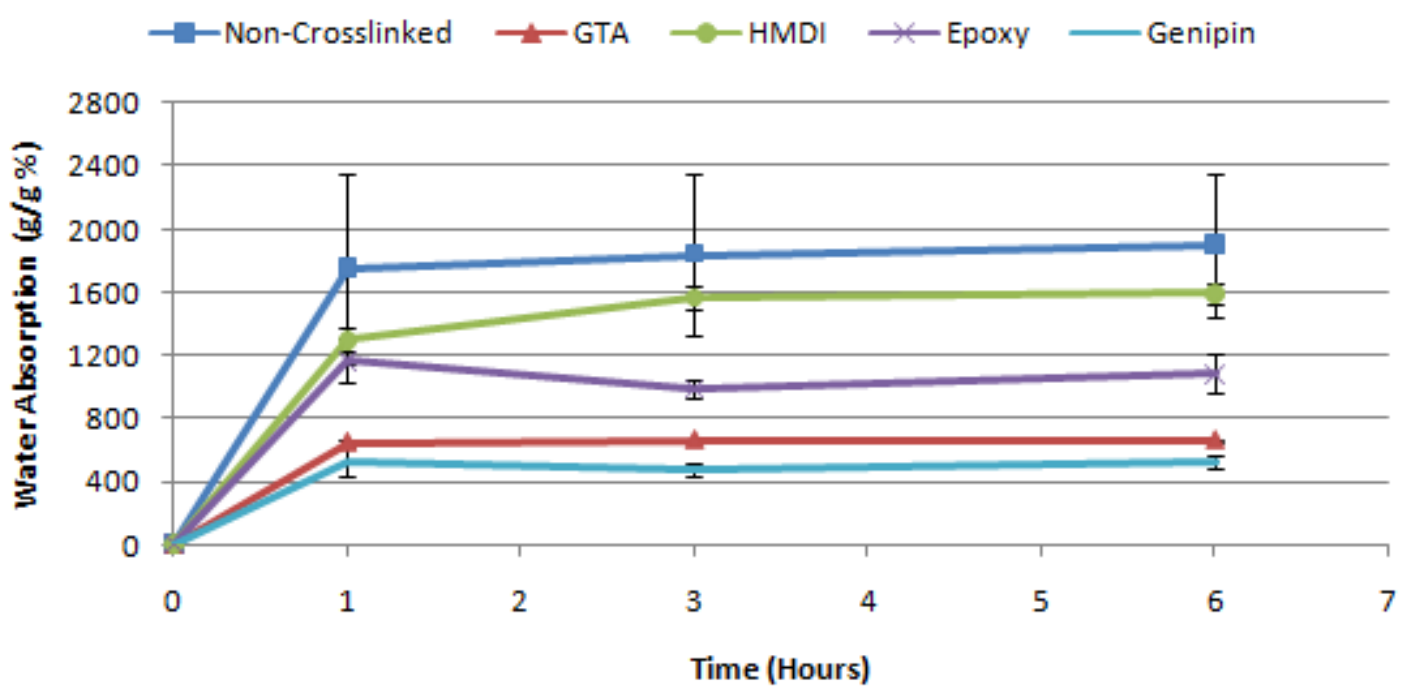

Figure 4: the swelling ratio of the scaffolds crosslinked with four different crosslinking agents as compared with non-crosslinked samples. Regardless of applied crosslinker, crosslinking reduced the swelling ratio of the scaffolds relative to the non-crosslinked samples. Genipin-crosslinked scaffolds showed the least swelling ratio and the HMDIcrosslinked samples showed the highest values amongst the crosslinked samples.

The swelling ratio of the scaffolds crosslinked with HMDI and epoxy was noticeably higher than the samples crosslinked with GTA and genipin. Aside from reduction in free amine groups, another reason for the reduction in the swelling ratio in the crosslinked samples is the configurational restrictions that occur in the tightly packed crosslinked structure [24]. As a result of covalent bond formation after crosslinking, the elasticity of the structure is reduced which hinders expansion of network in water and therefore constrains further water absorption. Therefore, crosslinking agents with longer molecular chains (such as HMDI and epoxy) would provide the structure with more ability to expand and swell in comparison with crosslinking agents with a shorter molecular chain length. This is reflected in the data reported in Figure 4. Genipin and GTA are believed to form a more compact structure than HMDI and epoxy which led to lower swelling ratio. When only genipin and GTA are compared the bulky heterocyclic structures of genipin, and its significantly shorter 
structure, is believed to cause a lower swelling ratio in genipin-crosslinked samples than the GTA-crosslinked samples [36].

\subsection{Microstructure Analysis}

Figure 5 displays the microstructure of porous gelatin scaffolds crosslinked with four crosslinking agents as compared with the non-crosslinked samples. The gas foaming method produced porous gelatin structures that had inter-connected pore systems. Such interconnectivity is critical for successful migration and proliferation of cells and the exchange of nutrients and waste products [37]. The average pore size of the crosslinked scaffolds with GTA, HMDI, Epoxy, and genipin are as follows: $226( \pm 66), 220( \pm 41), 389$ $( \pm 117), 520( \pm 163) \mu \mathrm{m}$, respectively. Smaller pore size of the HMDI-crosslinked scaffolds may be the result of propan-2-ol interaction with gelatin molecules during the crosslinking reaction. Alcohol solvents are shown to cause protein hydrophobicity, collapse, and shrinkage of molecular structure in an aqueous medium [38]. This may lead to shrinkage in the final structure. The microstructure of HMDI and epoxy-crosslinked scaffolds appeared to be smoother. It is shown that longer crosslinking agent molecules may increase the smoothness of the scaffold [16]. Among all crosslinked samples the genipin-crosslinked samples showed more distortion with the largest average pore size. Crosslinking gelatin scaffolds using genipin is reported to induce structural changes to the gelatin macromolecular structure [39]. This is attributed to the slow crosslinking kinetics of genipin relative to the faster rate of gelatin dissolution. 

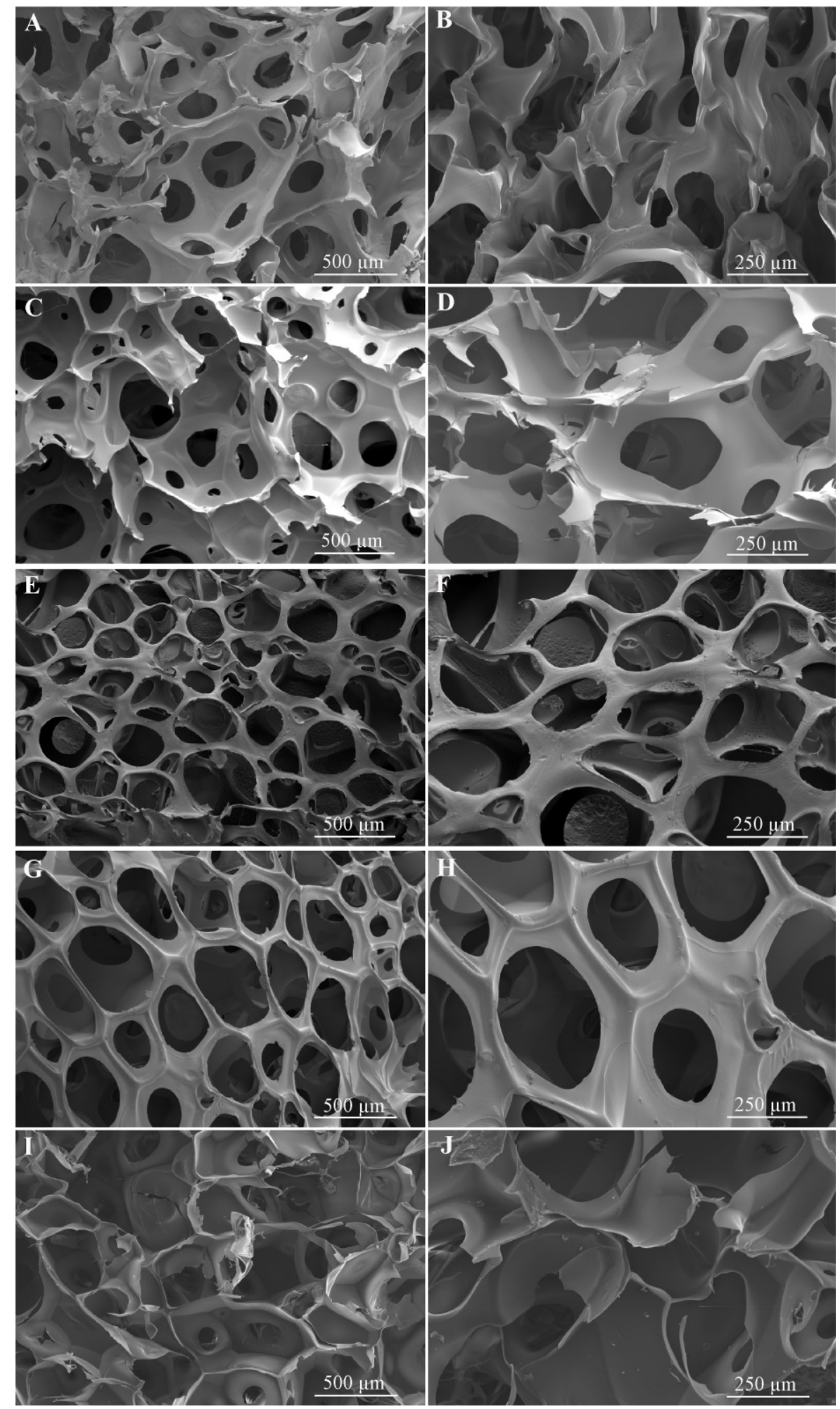

Figure 5: The microstructure of porous gelatin scaffolds crosslinked with four different crosslinking agents as compared with non-crosslinked samples at two magnifications. (A and B) non-crosslinked samples, ( $\mathrm{C}$ and D) GTA-crosslinked samples, (E and F) HMDIcrosslinked samples, ( $\mathrm{G}$ and $\mathrm{H}$ ) Epoxy-crosslinked samples, and (I and J) Genipincrosslinked samples. 


\subsection{Cell Culture Studies}

The viability of L929 fibroblast cells in contact with HMDI, genipin, GTA, and epoxycrosslinked scaffolds were quantified indirectly via the MTT assay and compared with noncrosslinked samples. The statistical analysis has been done at any given days among the crosslinked samples. After 1 day of cell seeding, there were no significant differences in cell viability among the crosslinked samples. However, on the $3^{\text {rd }}$ and $5^{\text {th }}$ post seeding days the GTA-crosslinked scaffolds showed a significantly lower biocompatibility in comparison the rest of crosslinked scaffolds $(\mathrm{p} \leq 0.05)$. On the $5^{\text {th }}$ day, the genipin-crosslinked scaffolds showed significantly higher biocompatibility than all other crosslinked samples $(p \leq 0.01)$ (Figure 6).

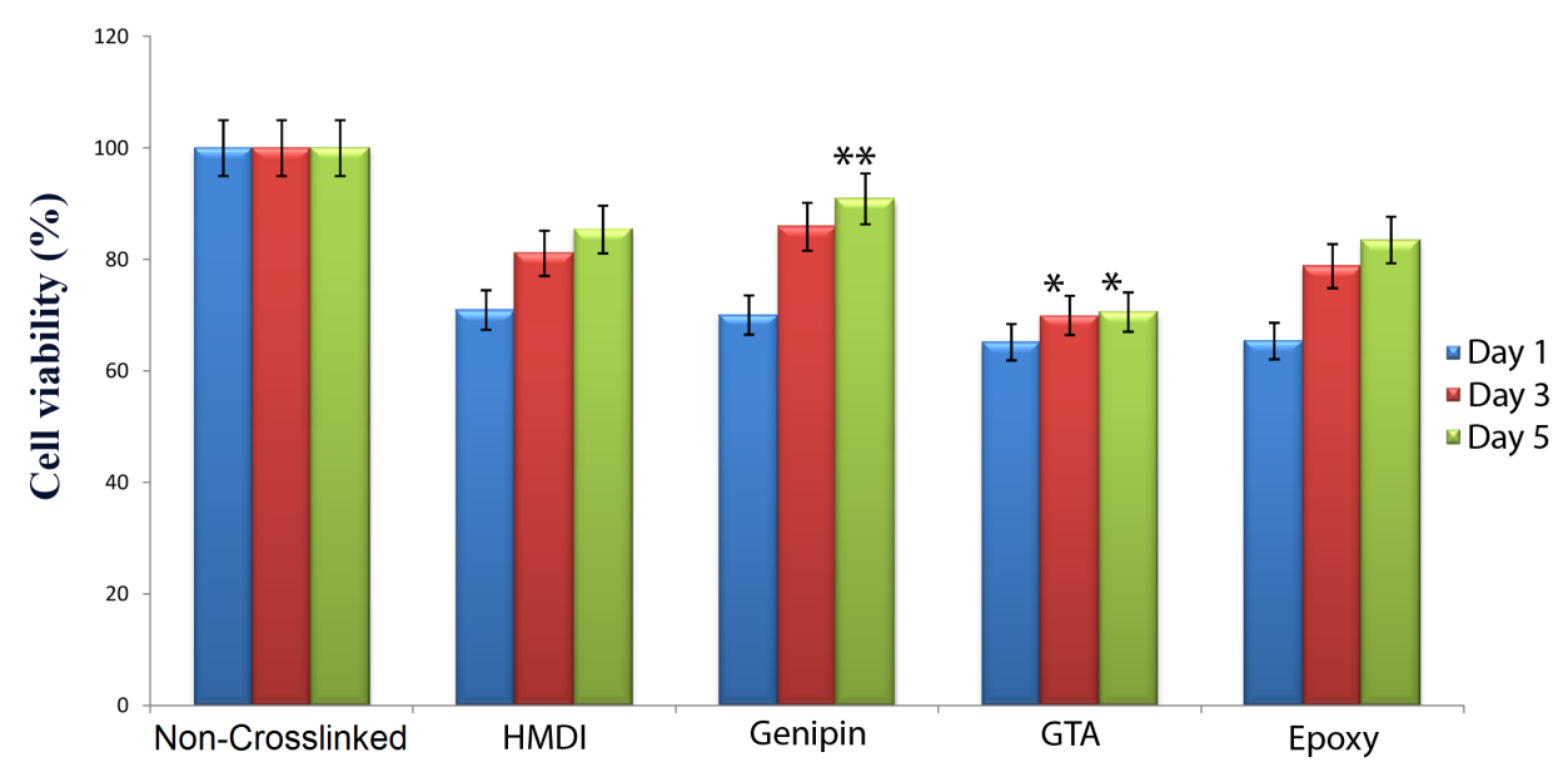

Figure 6: The results of MTT analysis on $1^{\text {st }}, 3^{\text {rd }}$, and $5^{\text {th }}$ post seeding days. The statistical analysis has been done at any given days among the crosslinked samples. On the first post seeding day there were no significant differences between the crosslinked samples. The cell viability for the GTA-crosslinked samples was significantly lower than the rest of samples on $3^{\text {rd }}$ and $5^{\text {th }}$ post seeding days $(* \mathrm{p} \leq 0.05)$. On the $5^{\text {th }}$ post seeding day the cell viability for the genipin-crosslinked scaffolds was significantly higher than the other samples $(* * p \leq 0.01)$.

Figure 7 shows the L929 cell proliferation pattern on the samples crosslinked by different crosslinking agents on the $5^{\text {th }}$ day of post seeding. It should be noted that the non- 
crosslinked samples did not remain intact for the 5 day cell culture period and incubation at $37^{\circ} \mathrm{C}$, due to the lack of thermal stability, and thus were not included in this test.

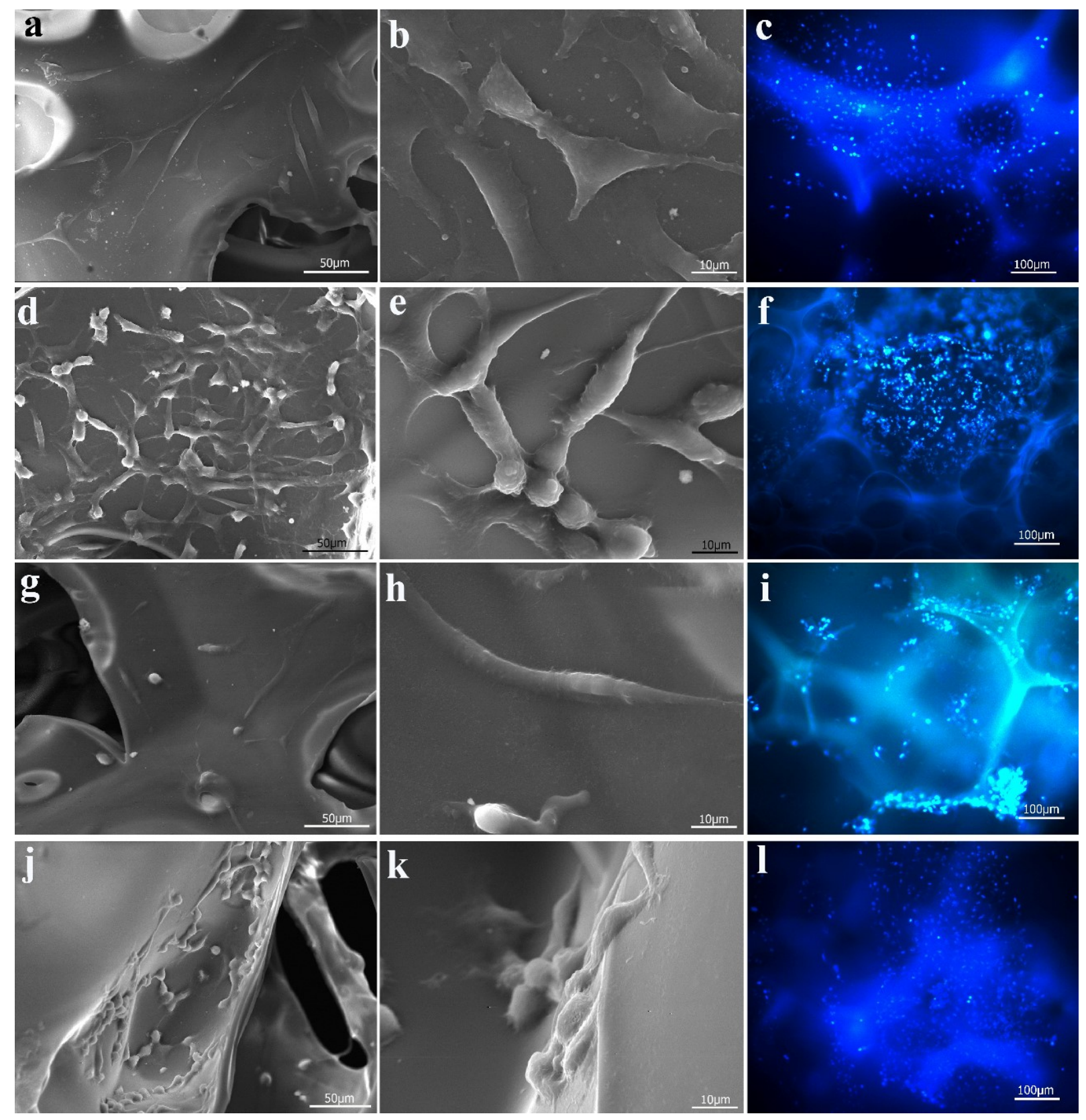

Figure 7: The cells spreading pattern as shown in SEM and DAPI staining analysis of the crosslinked scaffolds. (a-c) HMDI-crosslinked samples, (d-f) Genipin-crosslinked scaffolds, (g-i) GTA-crosslinked samples and (j-1) Epoxy-crosslinked samples. In a DAPI test, the cells nuclei are labelled in blue colour. Relatively more blue spots represent more viable cells on the scaffolds surface.

This observation indicates that after 5 days of cell culture, the cells maintained their adherence. However, relatively fewer cells were recognizable on the surface of GTAcrosslinked samples than the rest of crosslinked scaffolds. This was reaffirmed by DAPI 
staining and is in agreement with the MTT results which showed the lowest value of cell viability for the GTA-crosslinked scaffolds after 5 days of cell culture. Among HMDI, epoxy, and genipin-crosslinked scaffolds, the latter one could provide a better microenvironment as relatively more bright spots on the DAPI results represent more viable cells and cellular metabolism. Superior biocompatibility of genipin-crosslinked samples in comparison with synthetic crosslinking agents is reported by other researchers $[19,40]$.

\section{Conclusion}

As part of the previous report on the application of in-situ gas foaming, it was verified that crosslinking is an important step in this method. Here, a comparative study was reported with the aim of identifying a biocompatible and effective crosslinker for application in in-situ gas foaming. It was observed that longer molecules can lead to a more flexible structure with a lower Young's modulus and thermal stability but higher water absorption. The cytotoxicity analysis of this study reconfirmed the reported superior biocompatibility of genipin; however in term of pore structure, the genipin-crosslinked samples showed more distorted porosity. The other two non-GTA crosslinking agents that were used in this study (HMDI and epoxy) both produced structures with desirable microstructures and better biocompatibility relative to GTA. In summary, all three non-GTA alternative crosslinking agents that were used in this study showed promising potential for application in stabilizing porous gelatin scaffolds which are prepared via in-situ gas foaming. Based on the results of the current study HMDIcrosslinked samples demonstrated satisfying characteristics in all aspects of mechanical strength, microstructure, and cytotoxicity.

\section{Acknowledgements}


This work was financially supported in part by Armourers \& Brasiers' Gauntlet Trust (UK). SAP is grateful for the assistance from Mr. Mark A. Jones, while revising this manuscript.

\section{References}

[1] M.C. Gómez-Guillén, B. Giménez, M.E. López-Caballero, M.P. Montero, Functional and bioactive properties of collagen and gelatin from alternative sources: A review, Food Hydrocolloids. 25 (2011) 1813-1827.

[2] A. Barbetta, A. Gumerio, R. Pecci, R. Bedini, M. Dentini, Gas-in-Liquid Foam Templating as a Method for the Production of Highly Porous Scaffolds, Biomacromolecules. 10 (2009) 3188-3192.

[3] A. Poursamar, J. Hatami, A. Lehner, C. De silva, F. Ferreira, A.P.M. Antunes, Gelatin Porous Scaffolds Fabricated by a Simple Gas Foaming Technique: Characterisation and Cytotoxicity Assessment, Materials Science and Engineering: C. 48 (2015) 63-70.

[4] M. Azami, M. Rabiee, F. Moztarzadeh, Glutaraldehyde crosslinked gelatin/hydroxyapatite nanocomposite scaffold, engineered via compound techniques, Polymer Composites. 31 (2010) 2112-2120.

[5] G. Speit, S. Neuss, P. Schütz, M. Fröhler-Keller, O. Schmid, The genotoxic potential of glutaraldehyde in mammalian cells in vitro in comparison with formaldehyde, Mutation Research - Genetic Toxicology and Environmental Mutagenesis. 649 (2008) 146-154.

[6] R.A. Manji, A.H. Menkis, B. Ekser, D.K.C. Cooper, Porcine bioprosthetic heart valves: The next generation, American Heart Journal. 164 (2012) 177-185.

[7] W.D. Spotnitz, S. Burks, Hemostats, sealants, and adhesives: components of the surgical toolbox, Transfusion. 48 (2008) 1502-1516.

[8] S. Farris, J. Song, Q. Huang, Alternative reaction mechanism for the cross-linking of gelatin with glutaraldehyde, Journal of Agricultural and Food Chemistry. 58 (2010) 9981003.

[9] M.F. Butler, Y. Ng, P.D.A. Pudney, Mechanism and kinetics of the crosslinking reaction between biopolymers containing primary amine groups and genipin, Journal of Polymer Science Part A: Polymer Chemistry. 41 (2003) 3941-3953.

[10] D. Bellucci, A. Sola, P. Gentile, G. Ciardelli, V. Cannillo, Biomimetic coating on bioactive glass-derived scaffolds mimicking bone tissue, Journal of Biomedical Material Research Part A. 100A (2012) 3259-3266.

[11] D. Puppi, F. Chiellini, A.M. Piras, E. Chiellini, Polymeric materials for bone and cartilage repair, Progress in Polymer Science. 35 (2010) 403-440. 
[12] T.-. Chuang, M.-. Chen, F.-. Lin, Preparation and surface characterization of HMDIactivated 316L stainless steel for coronary artery stents, Journal of Biomedical Materials Research Part A. 85A (2008) 722-730.

[13] G. Dong, J. Sun, C. Yao, G.J. Jiang, C. Huang, F. Lin, A study on grafting and characterization of HMDI-modified calcium hydrogenphosphate, Biomaterials. 22 (2001) 3179-3189.

[14] J.B. Leach, J.B. Wolinsky, P.J. Stone, J.Y. Wong, Crosslinked $\alpha$-elastin biomaterials: towards a processable elastin mimetic scaffold, Acta Biomaterialia. 1 (2005) 155-164.

[15] A. Bigi, G. Cojazzi, S. Panzavolta, N. Roveri, K. Rubini, Stabilization of gelatin films by crosslinking with genipin, Biomaterials. 23 (2002) 4827-4832.

[16] G. Mabilleau, I.C. Stancu, T. Honoré, G. Legeay, C. Cincu, M.F. Baslé, D. Chappard, Effects of the length of crosslink chain on poly(2-hydroxyethyl methacrylate) (pHEMA) swelling and biomechanical properties, Journal of Biomedical Materials Research Part A. 77A (2006) 35-42.

[17] R.D. Patil, J.E. Mark, A. Apostolov, E. Vassileva, S. Fakirov, Crystallization of water in some crosslinked gelatins, European Polymer Journal. 36 (2000) 1055-1061.

[18] J. Nakka, K. Jansen, L. Ernst, Effect of chain flexibility in the network structure on the viscoelasticity of epoxy thermosets, Journal of Polymer Research. 18 (2011) 1879-1888.

[19] H. Liang, W. Chang, K. Lin, H. Sung, Genipin-crosslinked gelatin microspheres as a drug carrier for intramuscular administration: In vitro and in vivo studies, Journal of Biomedical Materials Research Part A. 65A (2003) 271-282.

[20] D.I. Zeugolis, G.R. Paul, G. Attenburrow, Cross-linking of extruded collagen fibers?A biomimetic three-dimensional scaffold for tissue engineering applications, Journal of Biomedical Materials Research Part A. 89A (2009) 895-908.

[21] L. Li, Y. Yu, Q. Wu, G. Zhan, S. Li, Effect of chemical structure on the water sorption of amine-cured epoxy resins, Corrosion Science. 51 (2009) 3000-3006.

[22] Q. Li, D. Yang, G. Ma, Q. Xu, X. Chen, F. Lu, J. Nie, Synthesis and characterization of chitosan-based hydrogels, International journal of biological macromolecules. 44 (2009) 121 127.

[23] J. Zhou, J.P. Lucas, Hygrothermal effects of epoxy resin. Part I: the nature of water in epoxy, Polymer. 40 (1999) 5505-5512.

[24] C.A. Miles, N.C. Avery, V.V. Rodin, A.J. Bailey, The Increase in Denaturation Temperature Following Cross-linking of Collagen is Caused by Dehydration of the Fibres, Journal of Molecular Biology. 346 (2005) 551-556.

[25] D. Porter, F. Vollrath, Water mobility, denaturation and the glass transition in proteins, Biochimica et Biophysica Acta (BBA) - Proteins and Proteomics. 1824 (2012) 785-791. 
[26] C.A. Miles, M. Ghelashvili, Polymer-in-a-Box Mechanism for the Thermal Stabilization of Collagen Molecules in Fibers, Biophysical journal. 76 (1999) 3243-3252.

[27] M. Catalina, G.E. Attenburrow, J. Cot, A.D. Covington, A.P.M. Antunes, Influence of crosslinkers and crosslinking method on the properties of gelatin films extracted from leather solid waste, Journal of Applied Polymer Science. 119 (2011) 2105-2111.

[28] R. Usha, T. Ramasami, Effect of crosslinking agents (basic chromium sulfate and formaldehyde) on the thermal and thermomechanical stability of rat tail tendon collagen fibre, Thermochimica Acta. 356 (2000) 59-66.

[29] C.Tonda-Turo, P. Gentile, S. Saracino, V. Chiono, V.K. Nandagiri, G. Muzio, R.A. Canuto, G. Ciardelli, Comparative analysis of gelatin scaffolds crosslinked by genipin and silane coupling agent, International Journal of Biological Macromolecules. 49 (2011) 700 706.

[30] K.J. Payne, A. Veis, Fourier transform ir spectroscopy of collagen and gelatin solutions: Deconvolution of the amide I band for conformational studies, Biopolymers. 27 (1988) 17491760 .

[31] M. Jackson, L. Choo, P.H. Watson, W.C. Halliday, H.H. Mantsch, Beware of connective tissue proteins: Assignment and implications of collagen absorptions in infrared spectra of human tissues, Biochimica et Biophysica Acta (BBA) - Molecular Basis of Disease. 1270 (1995) 1-6.

[32] J.H. Muyonga, C.G.B. Cole, K.G. Duodu, Fourier transform infrared (FTIR) spectroscopic study of acid soluble collagen and gelatin from skins and bones of young and adult Nile perch (Lates niloticus), Food Chemistry. 86 (2004) 325-332.

[33] F. Mi, H. Sung, S. Shyu, Synthesis and characterization of a novel chitosan-based network prepared using naturally occurring crosslinker, Journal of Polymer Science Part A: Polymer Chemistry. 38 (2000) 2804-2814.

[34] A. Kadnaim, W. Janvikul, U. Wichai, M. Rutnakornpituk, Synthesis and properties of carboxymethylchitosan hydrogels modified with poly(ester-urethane), Carbohydrate Polymers. 74 (2008) 257-267.

[35] F. Tasselli, A. Mirmohseni, M.S. Seyed Dorraji, A. Figoli, Mechanical, swelling and adsorptive properties of dry-wet spun chitosan hollow fibers crosslinked with glutaraldehyde, Reactive and Functional Polymers. 73 (2013) 218-223.

[36] H. Sung, D. Huang, W. Chang, R. Huang, J. Hsu, Evaluation of gelatin hydrogel crosslinked with various crosslinking agents as bioadhesives: In vitro study, Journal of Biomedical Materials Research. 46 (1999) 520-530.

[37] F. Dehghani, N. Annabi, Engineering porous scaffolds using gas-based techniques, Current opinion in biotechnology. 22 (2011) 661-666.

[38] B. Mohanty, H.B. Bohidar, Systematic of Alcohol-Induced Simple Coacervation in Aqueous Gelatin Solutions, Biomacromolecules. 4 (2003) 1080-1086. 
[39] S. Panzavolta, M. Gioffrè, M.L. Focarete, C. Gualandi, L. Foroni, A. Bigi, Electrospun gelatin nanofibers: Optimization of genipin cross-linking to preserve fiber morphology after exposure to water, Acta Biomaterialia. 7 (2011) 1702-1709.

[40] C. Tsai, R. Huang, H. Sung, H.C. Liang, In vitro evaluation of the genotoxicity of a naturally occurring crosslinking agent (genipin) for biologic tissue fixation, Journal of Biomedical Materials Research. 52 (2000) 58-65. 